Materials and Methods We conducted a retrospective observational study. We included all patients with MBC in 2007. Using the digital history, sociodemographic variables (age, sex), clinical (histologic type, oestrogen receptor, ER, progesterone receptor, PR, human epidermal growth factor receptor 2, HER-2, progression from primary tumour, appearance and location of metastases, lymph node involvement, survival, deceased) and therapeutic histories (radiotherapy, hormone therapy, chemotherapy) were collected.

Results We included 43 patients with a mean age of 54.5 years (100\% female). The most common histological types were infiltrating ductal (60\%) and lobular (24.4\%) tumours. ER and PR were positive in $75.6 \%$ and $50 \%$, respectively. Overexpression of Her- 2 was negative in $73.7 \%$. $69.2 \%$ of patients with MBC had progressed from primary tumour. The metastization appeared at an average of 44.1 months since diagnosis. The most common sites of metastases were bone (34.06\%), lung (16.48\%) and liver (20.86\%). $93.9 \%$ of patients had lymph node involvement and $77.6 \%$ were in the terminal phase. $95.3 \%$ of patients had received radiotherapy. Endocrine therapy used was fulvestrant (22.97\%), anastrozole $(21.62 \%)$, tamoxifen $(20.27 \%)$. All patients received chemotherapy, the most used first-line schemes being: epirubicin/cyclophosphamide/docetaxel (30.6\%), cyclophosphamide/epirubicin/fluorouracil $(20.4 \%)$ and cyclophosphamide/methotrexate/fluorouracil $(16.6 \%)$ in combination with trastuzumab or not. In successive lines, combinations of vinorelbine, docetaxel, capecitabine, carboplatin, gemcitabine were prescribed. Lapatinib and bevacizumab were used from the fourth-line treatment.

Conclusions Radiotherapy, not indicated in MBC, was used in early stages of the disease. Due to the variability of patients, treatment regimens are diverse and a predefined schema is not appropriate. Bevacizumab and lapatinib were used in late-stage treatment in patients who had not responded to standard treatment.

No conflict of interest.

\section{DGI-027 EFFECTIVENESS AND SAFETY OF CLOFARABINE IN PAEDIATRIC PATIENTS WITH ACUTE LEUKAEMIA}

doi:10.1136/ejhpharm-2013-000276.293

MC Garzas-Martin de Almagro, S De la Fuente Ruiz, N Garcia Melero, B Galvez Criado, T Brieva Herrera. Hospital Universitario Reina Sofia, Pharmacy, Cordoba, Spain

Background Clofarabine is a purine nucleoside antimetabolite, a second-generation antineoplastic indicated for the treatment of acute lymphoblastic leukaemia in paediatric patients ( $\leq 21$ years) who have relapsed or are refractory after receiving at least two prior regimens and who have no other treatment options that provide a durable response.

Despite progress in leukaemia treatment, most children who relapse have a dismal prognosis. New approaches are needed.

Purpose To assess the effectiveness and safety of clofarabine in paediatric patients with refractory or relapsed acute leukaemia.

Materials and Methods This was an observational retrospective study. We included all paediatric patients diagnosed with acute leukaemia who received clofarabine as antineoplastic treatment during 2007-2011.

We used the computer programme Oncofarm for prescribing, preparation and validation of chemotherapy treatments and collected data for number of patients, age, sex, weight, height and treatment schemes lines administered prior to clofarabine.

In addition, we used medical records as a source of safety data regarding potential adverse reactions due to clofarabine.

Results During the study period clofarabine was administered to a total of six paediatric patients ( 4 boys and 2 girls) with a median age of 11.5 years (5-16 years).
They had received a median of 2 prior lines of treatment. Later treatment schedules used in $83.3 \%$ of these patients included $40 \mathrm{mg} / \mathrm{m}^{2}$ clofarabine in combination with other chemotherapy drugs with a median of 2 administered cycles. In the rest (1/6) clofarabine was used at $52 \mathrm{mg} / \mathrm{m}^{2}$ as monotherapy.

$66.6 \%$ of patients achieved complete remission and $50 \%$ were transplanted.

Mucositis grade IV and pancytopenia were detected in two patients and palmar erythema in one patient. All patients had a transient transaminase increase.

Conclusions The administration of clofarabine allowed the team to reach transplantation in $50 \%$ of patients with acceptable toxicity, making it possible to expect durable responses.

No conflict of interest.

\section{DGI-028 EFFECTIVENESS AND SAFETY OF RITUXIMAB IN IDIOPATHIC THROMBOCYTOPENIC PURPURA}

doi:10.1136/ejhpharm-2013-000276.294

' $\mathrm{JJ}$ Elizondo, ${ }^{2} \mathrm{G}$ Elizondo, 'M Noceda, 'E Pellejero, 'M Elviro, 'M Gutierrez. ${ }^{1} \mathrm{CHN}$ Navarre Health Service, Pharmacy B, Pamplona, Spain; ${ }^{2}$ Navarre Health Service, Health Department, Pamplona, Spain

Background Idiopathic thrombocytopenic purpura (ITP) is an autoimmune disorder characterised by low platelet count and may be responsible for episodes of mucocutaneous bleeding of varying severity.

Purpose The study evaluates the effectiveness and safety of rituximab in patients who have not responded to first-line treatment.

Materials and Methods We performed a retrospective observational study, between 2009-2011, in adult patients who had not responded to first-line treatment (high-dose corticosteroids or non-specific IV immunoglobulins), or who were intolerant to such alternatives. Both splenectomised and non-splenectomised patients were included. The dose employed was $375 \mathrm{mg} / \mathrm{m}^{2} \mathrm{q} 7 \mathrm{~d}$ for four weeks. We considered it a partial response if the platelet count exceeded $50 \times 109$ cells/ $\mathrm{L}$, and a complete response if the count was greater than $100 \times 109$ cells/L. Previous duration of thrombocytopenia, platelet counts before treatment and after 4 weeks, percentage of patients having a satisfactory response, median time to response, duration, occurrence of petechiae and mucocutaneous bleeding and tolerability of the infusion were examined.

Results We recruited 22 patients, 12 men and 10 women. The mean age of the sample was 55.6 years (range: $19-88$ ) and a previous mean duration of ITP of 7.5 years (range 0.35 to 41 ). The mean platelet count before treatment was $32.8 \times 109$ cells/L (range: 6-70), and increased to $120 \times 109$ cells/L (range: 23-591) after completion within four weeks.

10 patients $(45.5 \%)$ experienced a complete response and in 8 patients (36.3\%) the response was partial, while 4 (18.2\%) patients experienced no response. The mean time to get some response was 2.3 weeks (range 1-4 weeks), and it continued a median of 10.5 months (range: $1-25)$. Among patients who had some response, in 2 cases petechiae and bleeding were detected again, while in 3 the platelet count fell below $50 \times 10^{9}$ cells $/ \mathrm{L}$. The other 13 patients who responded continue today with a platelet count within the target range and without clinical symptoms. The infusion was well tolerated in all cases.

Conclusions Rituximab seems an effective and well-tolerated alternative in patients with refractory ITP who require chronic treatment. This study and literature show that more than $50 \%$ of patients respond to treatment, and it may be an alternative to splenectomy. However, further prospective studies are required to define the optimal position of rituximab in the treatment of ITP.

No conflict of interest. 